\title{
Computer Assisted Three-dimensional Plaque Characterization in Intracoronary Ultrasound Studies
}

\author{
SA de Winter, I Heller, R Hamers, PJ de Feyter, PW Serruys, JRTC Roelandt, \\ N Bruining
}

Erasmus Medical Centre, Rotterdam, The Netherlands

\begin{abstract}
The volume, morphology and composition of atherosclerotic plaque play an important role in coronary artery disease and in long-term outcome of coronary interventions and pharmaceutical strategies. Intracoronary ultrasound (ICUS) provides real-time cross-sectional imaging of vessel wall structures. In-vitro and in-vivo studies have shown that ICUS can identify the general composition of the plaque by assigning different grey levels to different tissue types. Ultrasound scanners use 256 grey levels, whereas the human eye can only discriminate 32. Therefore, computer-assisted grey level interpretation (labelling) and measurement can help identify different tissue types present in the plaque and track changes over time. This paper covers a new research tool that facilitates this and that provides an objective quantitative analysis parameter for the segmentation of plaque into four different tissue types.
\end{abstract}

\section{Introduction}

Intracoronary ultrasound (ICUS) is used as a tomographic imaging technique to visualize the vessel wall morphology and to identify atherosclerotic plaque [1]. To examine a selected coronary vessel segment an ICUS imaging catheter is pulled back through the coronary artery, meanwhile acquiring a series of cross sectional images. Three-dimensional (3D) reconstruction of the segment and contour tracing in the ICUS images facilitate subsequent quantitative coronary ultrasound (QCU) analysis and qualifies ICUS to be used in clinical trials to evaluate the results of novel catheter-based interventional techniques as well as pharmaceutical treatments [2].

Knowledge of plaque composition is a valuable clinical parameter to assess information on plaque formation, progression and rupture [3] and plays an important role in gaining insight in the processes underlying events of sudden cardiac death [4]. Studies that compared histology to intravascular ultrasound learned that different structures correspond to different video-densities in the images; structures that appear white indicating high echogenicity, mainly consist of dense fibrous or calcific tissue. The darker, echolucent or hypoechogenic areas contain larger amounts of loose fibrous or smooth, muscle-rich tissue and thrombotic or necrotic elements [5]. Based on this idea, video-densitometry can be used for plaque characterization [7].

Manual classification of tissue in the ICUS images for an entire 3D segment is very time-consuming, and potentially error prone. The analyst has to judge the high number of ICUS images (typically 1500 images per minute pullback acquired at a speed of $0.5 \mathrm{~mm} / \mathrm{s}$ ) in a consistent way, which may result in large inter- and intraobserver variability. Moreover, the human eye can discriminate only about 32 levels of grey, while the ultrasound images can contain 256 levels. Computerassisted grey level interpretation (labelling) and measurement in an entire 3D segment can overcome these problems. Therefore we developed a method, based on video-densitometry, that can automatically label plaque tissue and quantify each labelled type in an existing ICUS pullback. This paper describes this method and shows how the results are presented and visualized in the new research tool.

\section{Methods}

ICUS pullbacks can be stored either on S-VHS videotape, or digitally on CD-ROM. Images originating from standard PAL S-VHS tapes are first de-interlaced to remove the effect of having duplicate images in one image frame. A Gaussian low-pass filter is applied to each cross section to smooth the effect of natural intensity fluctuations caused by the texture of that specific tissue. This ensures that echogenicity measurements focus on the identification of regions instead of individual pixels.

ICUS studies that are not acquired ECG-gated [8], are retrospectively image-based gated with the IntelliGate ${ }^{\circledR}$ $[13,14]$ method before QCU analysis. This allows accurate QCU results in anatomically correctly displayed segments. ECG -gating synchronizes the acquisition on the ECG signal from the patient during the intervention, while IntelliGate ${ }^{\circledR}$ does this synchronization afterwards 
based on information in the already acquired images.

\subsection{ICUS analysis}

In our method, QCU analysis is performed in longitudinal displays views (L-views) using the CURAD (Curad BV, Wijk bij Duurstede, Netherlands) analysis software [8] (see figure 1). In most cases only four Lviews, containing two contours per view, are needed for a complete analysis. Occasionally a fifth L-view with a $9^{\text {th }}$ contour is added to accommodate extreme eccentricity of the vessel. The analysis software is able to fully exploit automated contour detection algorithms in L-views to provide maximal reproducible analysis results with minimal intra- and inter-server variability. After performing QCU, the borders of the external elastic membrane (EEM) and the lumen enclose a volume that is used as a region of interest for plaque identification.

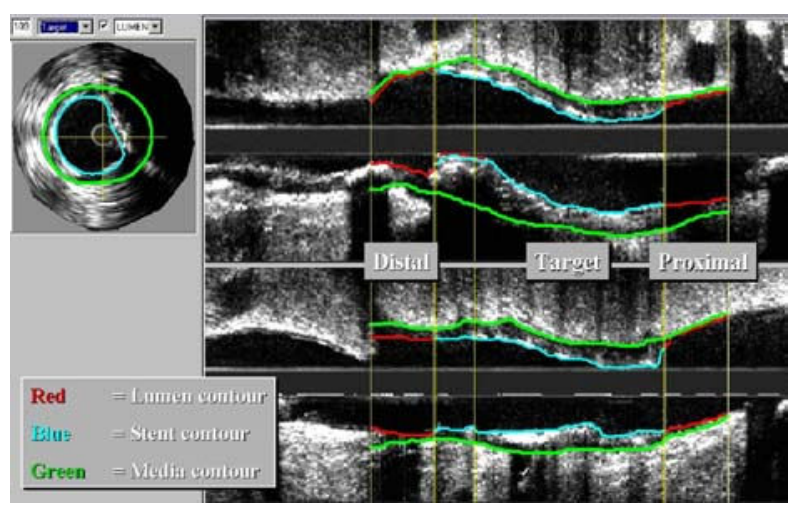

Figure 1. The Curad analysis software [8] allows contour tracing in the longitudinal views displayed on the right. Lumen and media contours are used to identify plaque. If available, a stent contour is used to define a region of interest for implanted stents.

\subsection{Characterization using echogenicity}

Unfortunately, there is no absolute classification map that prescribes which grey values in the plaque volume correspond to which tissue type. Different gain settings of ultrasound machines as well as different catheter types and machines [6] can cause the same structure appear in a different part of the grey spectrum.

To normalize for this behaviour the mean grey level of the adventita, located outside the EEM can be used as a discriminator between hypoechogenic and hyperechogenic tissue [3] (figure 2). Fibrous plaque can now be characterized as tissue that has a density that is similar or higher than that of the adventitia and hence will appear in grey levels that are mainly higher than that of the mean grey value of the adventitia [5-7]. Analogous, softer material has a lower grey value.

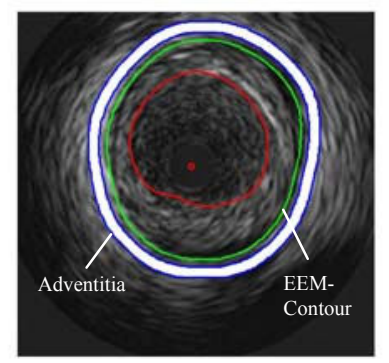

Figure 2. The adventitia is defined outside the EEM-contour.

In order to calculate the mean adventitia intensity (MAI), areas in the cross sections that represent shadow must be excluded to prevent a bias towards the dark part of the grey spectrum. Therefore, a rough estimate of shadow regions is made and excluded from further calculation. To examine the adventitia pixels for shadow, the image is divided into small 2 degree wide wedges that originate from the catheter center. In order to cover only true tissue data, the wedges are bounded either by the rectangular image boundary or by a user defined circular mask that indicates the area in the image where true echo data is present, whichever comes first. A radial scanning loop is used to judge all 180 wedges.

For all non-shadowed adventitia pixels, the mean value and standard deviation is calculated. To observe the suitability of the above mentioned clearing of shadow regions, a histogram of the residual adventitia pixels is created with an overlay of a standard normal probability distribution based on the the same mean and having the same standard deviation (figure 3). In case of a good match of histogram and standard normal curve, one can assume the MAI to be a correct parameter.

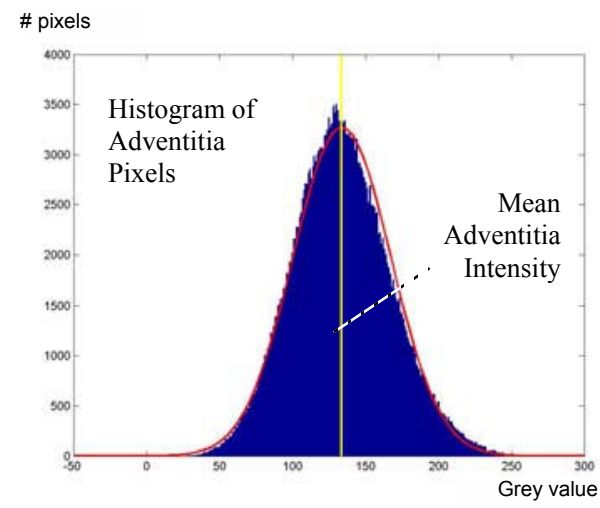

Figure 3. The adventitia pixels that remain after shadow exclusion follow a normal distribution.

The discrimination consists of the straightforward applying of the calculated MAI as a threshold to each cross-sectional image.

In addition, three other types can be identified as well: If acoustical shadow is present behind a highly echogenic 
spot, the tissue is likely to represent calcification. The highly echogenic parts are characterized as calcium. Although the shadowed parts also contain real tissue data, they are classified as unknown [7] tissue, since they are pictured in black in the image.

For the third tissue category, an upper discrimination threshold is set in addition to the MAI. This threshold value serves as an upper limit for the hyperechogenic region and is set to the MAI plus two times its standard deviation. All dense tissue that has a higher grey value than this threshold does not account for regular tissue, (stent-struts, calcifications with and without acoustical shadowing) and is characterized as 'upper' volume.

\section{Results}

QCU analysis provides all volumetric measurement results to define the region of interest for plaque characterization within the newly developed research tool. Both qualitative and quantitative results of the characterization process in the defined region are displayed in an interface as shown in figure 4 . For every cross section a histogram illustrates the grey level distribution of the plaque tissue in that image. In figure 4, panel A corresponds to the lowest part of the grey spectrum and contains all hypoechogenic regions. This tissue type area is separated from the hyperechogenic tissue B by the grey value of the adventitia. Very bright grey values higher than the upper discrimination value are shown in $\mathrm{C}$. The results of the shadow exclusion for the calculation of the MAI are shown in the upper left cross section of figure 4 .
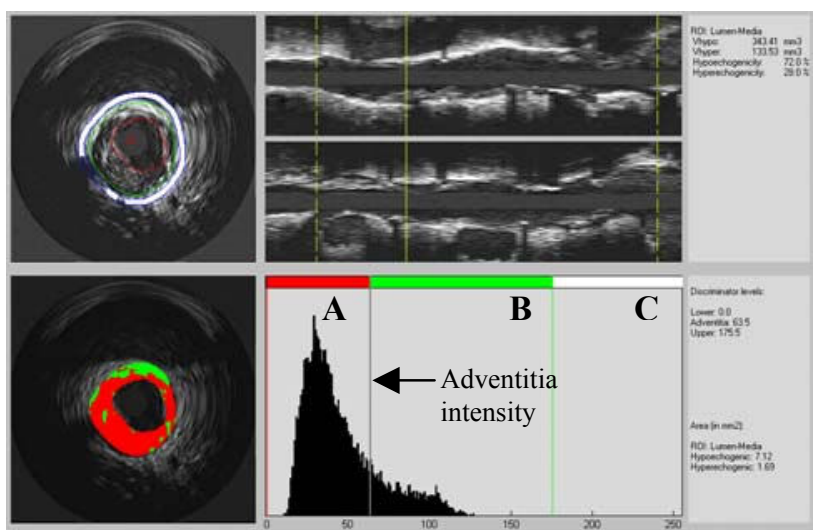

Figure 4. Picture of the interface used for plaque characterization. On the upper left panel the adventitia with the shadow parts excluded is shown. The right image shows a longitudinal view that is also used for navigation through the set. The lower panel shows the same cross section, with the result of the color labelling overlaid. The majority of the plaque is located in A and is therefore hypoechogenic.
Every cross section can be inspected individually and the location of the different tissue types is visualized as a color overlay shown in the lower cross section, next to the histogram in figure 4.

The quantitative results are stored in a separate report. Separate volumes are presented for: hyperechogenic, hypoechogenic, 'upper', calcium, unknown tissue and total plaque volume. Plaque tissue volume is expressed as that part of the region of interest that can be classified as either hyperechogenic or hypoechogenic tissue. The calcium, unknown and upper volume are presented separately, although they may overlap each other, as is the case with the upper volume that will include calcium spots as well. For every tissue type the percentage of the total volume is also displayed to allow easy comparison in the case of baseline and follow-up studies for the same patient.

As an additional visual aid, the plaque volume can be reconstructed as a rendered $3 \mathrm{D}$ object, which colorlabels hyperechogenic volumes in green and the hypoechogenic ones in red. (figure 5).

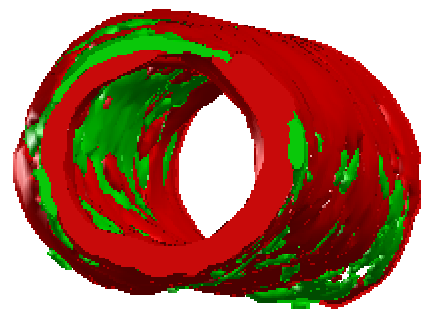

Figure 5. 3D representation of the plaque volume with the hyperechogenic and hypoechogenic volumes colorlabelled. The darker red structures are hypoechogenic and the lighter green spots are hyperechogenic.

\section{Discussion}

It is difficult to manually assess video-densitometry measurements on a large number of ICUS images and nearly all studies use only a few frames per patient. However, evaluation within our tool learned that plaque composition differs largely from frame to frame. Automated grey level interpretation can produce consistent results and facilitates the examination of longer segments with video-densitometry. This also opens a new perspective on the evaluation of progressionregression studies. Plaque characterization can now easily be done in the same segment at baseline and follow-up.

Our method uses gated pullbacks to obtain good QCU results and to ensure anatomical correctness, which is important since the volumetric measurements in the plaque characterization tool depend on a proper 3D reconstruction.

Image segmentation has to be performed before the adventitia can be found. Some studies demonstrated that spectral analysis of the radiofrequency (RF) data from the 
ultrasound signal has the potential to perform highresolution tissue characterization in real time $[10,11]$ and suggest that grey level analysis is not reliable in detecting heterogeneous tissue areas [12]. However, the acquisition and analysis of the large amount of RF data requires fast and expensive hardware. In contrast, our research tool can be operated on standard workstations. Moreover, RF analysis can only be done prospectively and cannot be used to compare findings with those in existing studies. Resolution of the backscatter signal is high, which would be beneficial in vulnerable plaque detection. Unfortunately, the identification of affected lesions with ultrasound in general is very difficult and may require new catheters before the high-resolution signal can be fully understood in relation to histological data.

\section{Limitations}

Grey level mapping and its dynamic range are dependent on the abilities of the video conversion systems in the ultrasound machines. Nevertheless, our histogram showed a good distribution in $94 \%$ of 68 randomly selected analogous tapes from one study.

Currently, the presented method is being validated invitro. Histological correlation with our ultrasound findings will quantify the discrimination performance of the tool and might give rise to optimize the characterization process further and/or proves its correctness.

\section{Conclusion}

In conjunction with proper QCU analysis software, computer assisted interpretation of grey levels and measurement in ultrasound images can contribute to the assessment of qualitative and quantitative information on the plaque composition of analyzed 3D coronary segments. Preliminary evaluation showed good results and initiated an in-vitro validation procedure.

\section{References}

[1] Yock PG, Linker DT. Intravascular ultrasound. Looking below the surface of vascular disease. Circulation 1990;81(5):1715-8.

[2] Nissen SE, Yock P. Intravascular ultrasound: novel pathophysiological insights and current clinical applications. Circulation 2001;103(4):604-16.

[3] Okimoto T, Imazu M, Hayashi Y, Fujiwara H, Ueda H, Kohno N. Atherosclerotic plaque characterization by quantitative analysis using intravascular ultrasound: correlation with histological and immunohistochemical findings. Circ J 2002;66(2):173-7.

[4] Virmani R, Kolodgie FD, Burke AP, Farb A, Schwartz SM. Lessons from sudden coronary death: a comprehensive morphological classification scheme for atherosclerotic lesions. Arterioscler Thromb Vasc Biol 2000;20(5):1262-
75.

[5] Prati F, Arbustini E, Labellarte A, Dal Bello B, Sommariva L, Mallus MT, et al. Correlation between high frequency intravascular ultrasound and histomorphology in human coronary arteries. Heart 2001;85(5):567-70.

[6] Hiro T, Leung CY, Russo RJ, Moussa I, Karimi H, Farvid $\mathrm{AR}$, et al. Variability in tissue characterization of atherosclerotic plaque by intravascular ultrasound: a comparison of four intravascular ultrasound systems. Am J Card Imaging 1996;10(4):209-18.

[7] Schartl M, Bocksch W, Koschyk DH, Voelker W, Karsch $\mathrm{KR}$, Kreuzer J, et al. Use of intravascular ultrasound to compare effects of different strategies of lipid-lowering therapy on plaque volume and composition in patients with coronary artery disease. Circulation 2001;104(4):387-92.

[8] Hamers R, Bruining N, Knook M, Sabate M, Roelandt JRTC. A Novel Approach to Quantitative Analysis of Intra Vascular Ultrasound Images. In: Murray A, editor. Computers in Cardiology; 2001 Sep 23-26; Rotterdam, The Netherlands: IEEE; 2001. p. 589-592.

[9] Bruining N, von Birgelen C, de Feyter PJ, Ligthart J, Li W, Serruys PW, et al. ECG-gated versus nongated threedimensional intracoronary ultrasound analysis: implications for volumetric measurements. Cathet Cardiovasc Diagn 1998;43(3):254-60.

[10] Nair A, Kuban BD, Tuzcu EM, Schoenhagen P, Nissen SE, Vince DG. Coronary plaque classification with intravascular ultrasound radiofrequency data analysis. Circulation 2002;106(17):2200-6.

[11] Moore MP, Spencer T, Salter DM, Kearney PP, Shaw TR, Starkey IR, et al. Characterisation of coronary atherosclerotic morphology by spectral analysis of radiofrequency signal: in vitro intravascular ultrasound study with histological and radiological validation. Heart 1998;79(5):459-67.

[12] Palmer ND, Northridge D, Lessells A, McDicken WN, Fox KA. In vitro analysis of coronary atheromatous lesions by intravascular ultrasound; reproducibility and histological correlation of lesion morphology. Eur Heart J 1999;20(23):1701-6.

[13] de Winter SA, Hamers R, Degertekin M, Tanabe K, Lemos PA, Serruys PW, Roelandt JRTC, Bruining N. Retrospective Image-Based Gating of Intracoronary Ultrasound Images for Improved Quantitative Analysis: The Intelligate Method Cath. And Cardiovasc. Interventions. In press.

[14] de Winter SA, Hamers R, Degertekin M, Tanabe K, Lemos PA, Serruys PW, Roelandt JRTC, Bruining N. A Novel Retrospective Gating Method for Intracoronary Ultrasound Images based on Image Properties. In: Computers in Cardiology; 2003 Sep 21-24; Thessaloniki, Greece: IEEE; 2003. In press.

Address for correspondence.

SA de Winter

Erasmus Medical Centre/Room H553

Dr. Molewaterplein 40

3015 GD Rotterdam

The Netherlands

E-mail: s.dewinter@erasmusmc.nl 